\title{
Two equivalent multi-sensor Kalman filters with variable delays and intermittent measurements
}

\author{
Babak Tavassoli, Parisa Joshaghani
}

\begin{abstract}
Kalman filtering of measurement data from multiple sensors with time-varying delays and missing measurements is considered in this work. Two existing approaches to Kalman filtering with delays are extended by removing some assumptions in order to have equivalent filtering methods and making comparisons between them. The computational loads of the two methods are compared in terms of the average number of floating point operations required by each method for different system dimensionalities and delay upper bounds. The results show that the superiority of the methods over each other depends on the comparison conditions.
\end{abstract}

Index Terms-Kalman filter, time-varying delays, intermittent measurements, data packet losses, multirate sampling.

\section{INTRODUCTION}

There is a wide variety of filtering applications where timevarying delays, missing measurement samples, and multiplicity of sensors are issues in many of them. Delays and missing measurements can be for example due to processing time or communication constraints [1], [2]. Kalman filtering with only random data packet losses is considered in a number of works including [3], [4], [5], [6] where the dimensionality of filter is the same as the dynamical system. However, the filter becomes more complex in the presence of delays.

There are three main approaches to filter design with delayed measurement information. The first approach is to revisit the past values of the recursive Kalman filtering variables upon receiving a delayed measurement sample. To reduce the complexity of this approach, the covariance matrix is calculated regardless of delay occurences in [7], [8] which causes some estimation errors. These error can be avoided by storing the past filtering variables in finite length buffers as suggested in [9] where data packet losses are also taken into account. Some researchers attempt to combine the recursive filter equations for the previous time steps insteed of using buffers which can increase the complexity of filter [7], [10], [11]. This complexity can be reduced to some extend by making simplifying assumptions for example about order or availability of data samples in [12], [13], [14], [15]. The second approach is based on augmentation of the state vector with the delayed measurements. This converts the delayed system to a delay-free system such that the ordinary Kalman filter can be applied [2], [16], [17], [7]. The third approach applies $H_{\infty}$ analysis of time delay systems to design filters with bounded error covariance [18], [19], [20], [21]. The result is not a Kalman filter in the sense that it does not produce the

B. Tavassoli is with the K.N. Toosi University of Technology, Tel.: +9821-88462174, Fax: +98-21-88462066, (tavassoli@kntu.ac.ir).

P. Joshaghani was with the K.N. Toosi University of Technology. optimal estimation. It is relatively straightforward to design robust filters based on this approach [22], [23], [24].

This work aims to extend the first two Kalman filtering approaches mentioned above into equivalent methods such that a comparison between them is made possible. The extensions allow for tackling intermittent measurements from multiple sensors subject to time-varying delays. For the first approach, the filtering method in [9], which is found to be a more general formulation among the existing results, is extended to the case of multi-sensor measurements while modifying the underlying notation. The result is presented as a selfcontained optimal filtering algorithm. The formulation of the second approach is also extended by adding the capabilities to handle missing measurements and simultaneous or missordered arrival of measurement samples from the previous time steps. Then, the computational loads of the two methods are compared for different values of delay upper bound and system's dimentionality by evaluating the average count of floating point operations (flops) required for each case. The results show that each method can overtake the other one in a subset of conditions.

Notation: The set of integers $\{a, a+1, \cdots, b\}$ is denoted by $\{a . . b\}$. For an ordered set $S$, a set of elements $M_{i}$ for $i \in S$ is denoted as $\left\{M_{i}\right\}_{i \in S}$ or $\left\{M_{i}\right\}_{i=a}^{b}$ if $S=\{a . . b\}$. Vertical and diagonal concatenations of matrices $\left\{M_{i}\right\}_{i=a}^{b}$ are denoted by cat $\left\{M_{i}\right\}_{i=a}^{b}$ and $\operatorname{diag}\left\{M_{i}\right\}_{i=a}^{b}$ respectively. The expected value of a random matrix $V$ is denoted by $\mathbb{E}\{V\}$. A matrix with zero elements is simply denoted as 0 if its dimensions can be inferred from the containing formula. An empty matrix is denoted by $0_{0}$ which is allowed to be concatenated with an arbitrary matrix $M$ as cat $\left\{M, 0_{0}\right\}=\operatorname{diag}\left\{M, 0_{0}\right\}=M$.

\section{Problem Statement}

The problem under consideration in this work is to optimally estimate the state of a dynamical system described by

$$
\begin{aligned}
x_{k} & =F_{k} x_{k-1}+B_{k} u_{k}+w_{k} \\
y_{k}^{i} & =H_{k}^{i} x_{k}+v_{k}^{i}
\end{aligned}
$$

in which $k$ is the discrete time step, $x_{k} \in \mathbb{R}^{n_{x}}$ is the state vector, $u_{k} \in \mathbb{R}^{n_{u}}$ is the input vector, $y_{k}^{i} \in \mathbb{R}^{n_{i}}$ is the $i$ th measured output vector for $i \in\{1 . . m\}$ with $\sum_{i=1}^{m} n_{i}=n_{y}, w_{k} \in \mathbb{R}^{n_{w}}$ and $v_{k}^{i} \in \mathbb{R}^{n_{i}}$ are uncorrelated, zero-mean white Gaussian noise vectors with covariance matrices $R_{k}^{i}=\mathbb{E}\left\{v_{k}^{i} v_{k}^{i T}\right\}$ and $Q_{k}=\mathbb{E}\left\{w_{k} w_{k}^{T}\right\}$.

The $i$ th output sample $y_{k}^{i}$ is received by the estimator after a time-varying delay defined as

$$
d_{k}^{i}=t-k \quad \text { if the estimator receives } y_{k}^{i} \text { at } t \geq k .
$$


It is assumed that the measurement samples are timestamped such that, the delay for each data sample can be calculated from its time-stamp by the estimator. Missing measurements are also assumed to be probable for example due to multirate sampling or communication errors. A missing measurement can be considered as a sample received with infinite delay. If a measurement sample is not missed, it is assumed to be received after a delay bounded by $d_{\max }$ such that

$$
d_{k}^{i} \in\left\{0 . . d_{\max }\right\} \cup\{\infty\} \quad \forall k \geq 0
$$

The optimal estimation of the state $x_{k}$ is given by

$$
\begin{aligned}
\hat{x}_{k \mid k} & =\mathbb{E}\left\{x_{k} \mid \mathcal{I}_{k}\right\} \\
\mathcal{I}_{k} & =\left\{y_{j}^{i} \mid j+d_{j}^{i} \leq k\right\}
\end{aligned}
$$

in which $\mathcal{I}_{k}$ is the information set which is available to the estimator at the time step $k$.

If there are no delays or missing measurements (i.e. $d_{k}^{i}=0$ ), then the optimal estimation $\hat{x}_{k \mid k}$ is calculated by the means of the ordinary Kalman filter in the appendix. In presence of the delays or missing measurements, the ordinary Kalman filter cannot be applied. In the following two sections, two equivalent approaches are presented for extension of Kalman filtering to measured data from multiple sensors, with variable delays and missing measurements according to (3).

Remark 1: The problem formulation in this section is capable for tackling multirate sampling systems. For this purpose, it is only needed to virtually assume that $d_{k}^{i}=\infty$ if no sample is taken from the $i$ th sensor at the time step $k$.

\section{BACKWARD RENOVATION KALMAN FILTER}

In the first method, if $y_{k}^{i}$ is received at the time step $t$, then the state estimator has to repeat the recursive calculations of filter from $k$ to $t$. To formulate this method, the following notation is introduced for the variables $y_{k}^{i}, v_{k}^{i}, H_{k}^{i}, R_{k}^{i}$.

$$
\begin{aligned}
& \tilde{\varphi}_{k}^{i, t}= \begin{cases}\varphi_{k}^{i} & \text { if } y_{k}^{i} \text { is received until } t, \\
0_{0} & \text { otherwise, }\end{cases} \\
& \varphi \in\{y, v, H, R\} .
\end{aligned}
$$

The set of measurements that are sampled at $k$ and received until $t$ are concatenated into a vector $\tilde{y}_{k}^{t}$ defined as

$$
\tilde{y}_{k}^{t}=\operatorname{cat}\left\{\tilde{y}_{k}^{i, t}\right\}_{i=1}^{m}
$$

which can be written as

$$
\begin{aligned}
\tilde{y}_{k}^{t} & =H_{k}^{t} x_{k}+\tilde{v}_{k}^{t}, \\
H_{k}^{t} & =\operatorname{cat}\left\{\tilde{H}_{k}^{i, t}\right\}_{i=1}^{m}, \\
\tilde{v}_{k}^{t} & =\operatorname{cat}\left\{\tilde{v}_{k}^{i, t}\right\}_{i=1}^{m},
\end{aligned}
$$

in which $\tilde{v}_{k}^{t}$ is a new zero-mean noise vector with covariance

$$
R_{k}^{t}=\mathbb{E}\left\{\tilde{v}_{k}^{t} \tilde{v}_{k}^{t^{T}}\right\}=\operatorname{diag}\left\{\tilde{R}_{k}^{i, t}\right\}_{i=1}^{m} .
$$

Defining the information set $\mathcal{I}_{k}^{t}$ as

$$
\mathcal{I}_{k}^{t}=\left\{\tilde{y}_{j}^{i, t} \mid j \leq k, 1 \leq i \leq m\right\},
$$

then we have $\mathcal{I}_{k}^{k}=\mathcal{I}_{k}$ according to (4b) and (5) for $\varphi=y$.
The optimal estimation of $x_{k}$ in (24) and the corresponding error covariance given the information set $\mathcal{I}_{h}^{t}$ for time steps $t$ and $h$ satisfying $t \geq k \geq h$ are written as

$$
\begin{aligned}
& \hat{x}_{k \mid h}^{t}=\mathbb{E}\left\{x_{k} \mid \mathcal{I}_{h}^{t}\right\} \\
& P_{k \mid h}^{t}=\mathbb{E}\left\{\left(x_{k}-\hat{x}_{k \mid h}^{t}\right)\left(x_{k}-\hat{x}_{k \mid h}^{t}\right)^{T} \mid \mathcal{I}_{h}^{t}\right\}
\end{aligned}
$$

Since $\mathcal{I}_{k}^{k}=\mathcal{I}_{k}$, the optimal estimation in (4) satisfies

$$
\hat{x}_{k \mid k}=\hat{x}_{k \mid k}^{k}
$$

For a given time step $t$, the ordinary Kalman filer equations in the appendix can be applied to write

$$
\begin{aligned}
& \hat{x}_{k \mid k-1}^{t}=F_{k} \hat{x}_{k-1 \mid k-1}^{t}+B_{k} u_{k} \\
& P_{k \mid k-1}^{t}=F_{k} P_{k-1 \mid k-1}^{t} F_{k}^{T}+Q_{k} \\
& K_{k}^{t}=P_{k \mid k-1}^{t} H_{k}^{t^{T}}\left(H_{k}^{t} P_{k \mid k-1}^{t} H_{k}^{t^{T}}+R_{k}^{t}\right)^{-1} \\
& \hat{x}_{k \mid k}^{t}=\hat{x}_{k \mid k-1}^{t}+K_{k}^{t}\left(\tilde{y}_{k}^{t}-H_{k}^{t} \hat{x}_{k \mid k-1}^{t}\right) \\
& P_{k \mid k}^{t}=\left(I-K_{k}^{t} H_{k}^{t}\right) P_{k \mid k-1}^{t} .
\end{aligned}
$$

By the definition in (5), the variables $\tilde{\varphi}_{k}^{i, t}$ for $\varphi \in\{y, H, R\}$ remain constant with respect to $t$ if $t \geq k+d_{\text {max }}$. Hence, the equations (12) are the same for $t \geq k+d_{\max }$ such that

$$
k<t-d_{\text {max }} \Longrightarrow\left\{\begin{array}{l}
\hat{x}_{k \mid k}^{t}=\hat{x}_{k \mid k}^{t-1} \\
P_{k \mid k}^{t}=P_{k \mid k}^{t-1}
\end{array} .\right.
$$

Therefore, at every time step $t$ the estimator only needs to recalculate $\hat{x}_{k \mid k}^{t}$ and $P_{k \mid k}^{t}$ for $k \in\left\{t-d_{\max } . . t\right\}$ by repeating the recursive filtering calculations in (12). For this purpose, it is needed that the estimator is equipped with data buffers of length $d_{\max }$ to store $\hat{x}_{k \mid k}^{t}, P_{k \mid k}^{t}$ for $k \in\left\{t-d_{\max } . t\right\}$.

The estimator also needs to keep track of $y_{k}^{i}, v_{k}^{i}, H_{k}^{i}, R_{k}^{i}$ to update $\tilde{\varphi}_{k}^{i, t}$ in (5) for $\varphi \in\{y, H, R\}$ that are required for repeating the recursive calculations. For this purpose, the set $\mathcal{J}_{t}$ which indexes the samples received at $t$ is defined as

$$
\mathcal{J}_{t}=\left\{(j, i) \mid d_{t-j}^{i}=j\right\}
$$

which according to (3) satisfies

$$
\mathcal{J}_{t} \subseteq\left\{0 . . d_{\max }\right\} \times\{1 . . m\} .
$$

The equations in this section can be converted to the Algorithm 1 which gets the newly arrived information at every time step $t$ given by $\left\{y_{t-j}^{i}, H_{t-j}^{i}, R_{t-j}^{i}\right\}_{(j, i) \in \mathcal{J}_{t}}$ and calculates $\hat{x}_{t \mid t}$ in (4). For this purpose, the algorithm stores $\hat{x}_{k \mid k}^{t}$ and $P_{k \mid k}^{t}$ for $k \in\left\{t-d_{\max } . . t\right\}$ in buffers $\bar{x}, \bar{P}$ with finite length $\ell \in\left\{0 . . d_{\max }\right\}$. The additional buffers $\bar{y}, \bar{H}, \bar{R}$ are also used to store the received samples of $y_{k}^{i}$ and the corresponding $H_{k}^{i}$ and $R_{k}^{i}$ for $k \in\left\{t-d_{\text {max }} . . t\right\}$ and $i \in\{1 . . m\}$. These value are used for repeating the recursive filter calculations between lines 8 and 13 of the algorithm. The non-stored auxiliary variables are denoted using non-italic names. In particular, $x$ and $P$ stand for $\hat{x}_{k \mid k-1}^{t}$ and $P_{k \mid k-1}^{t}$. The value of $\ell$ starts from zero and grows step by step up to $d_{\max }$ after which the buffer lengths remain constant. At $t=0$ the buffers must be initialized as

$$
\begin{aligned}
& \left(\ell, \bar{x}_{0}, \bar{P}_{0}\right) \leftarrow\left(0, \hat{x}_{0}, P_{0 \mid 0}\right) \\
& \left(\bar{y}_{0}^{i}, \bar{H}_{0}^{i}, \bar{R}_{0}^{i}\right) \leftarrow\left(0_{0}, 0_{0}, 0_{0}\right) \quad \forall i \in\{1 . . m\}
\end{aligned}
$$




$$
\begin{aligned}
& 1 \text { function } \operatorname{BRF}\left(t, \mathcal{J}_{t},\left\{y_{t-j}^{i}, H_{t-j}^{i}, R_{t-j}^{i}\right\}_{(j, i) \in \mathcal{J}_{t}},\right. \\
& \left.\qquad, \bar{x}_{0 . . \ell}, \bar{P}_{0 . . \ell}, \bar{y}_{0 . . \ell}^{1 . . m}, \bar{H}_{0 . . \ell}^{1 . . m}, \bar{R}_{0 . . \ell}^{1 . . m}\right)
\end{aligned}
$$

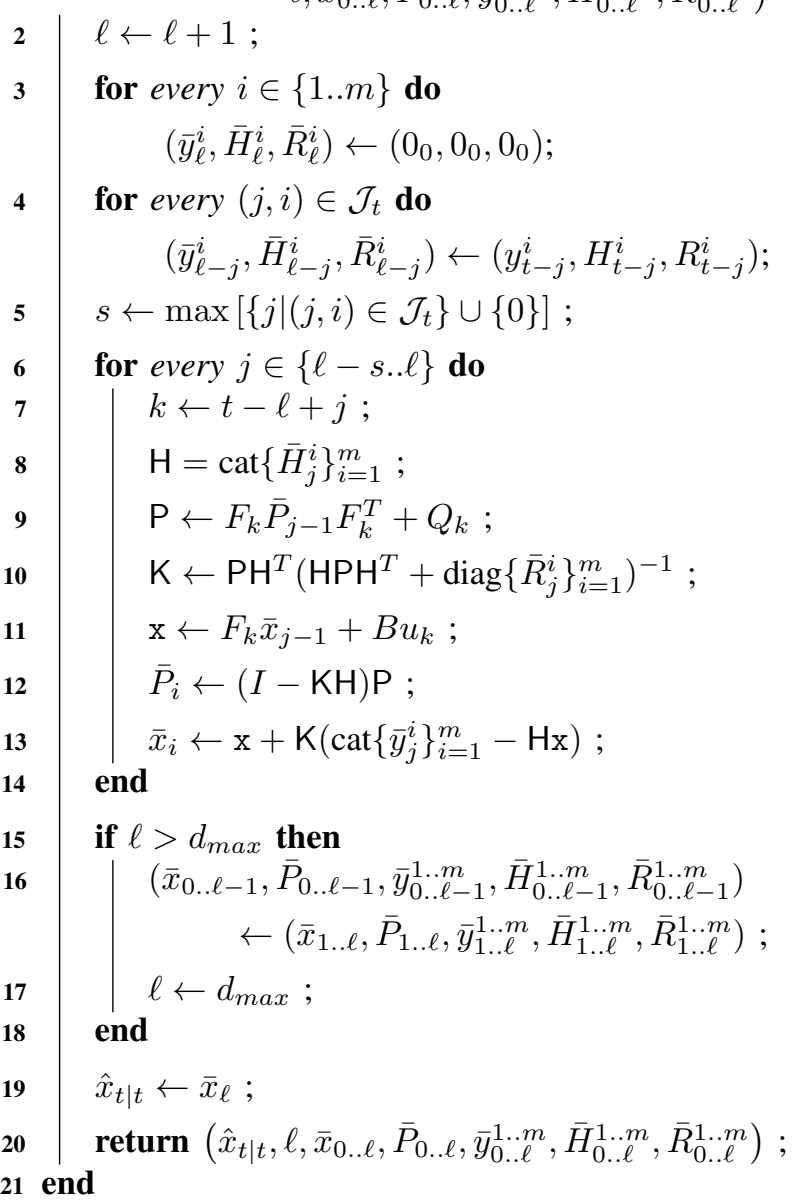

Algorithm 1: Backward renovation filtering algorithm

\section{Augmented System FILtering}

In the second method for obtaining the estimation of state in (4), the system (24) is augmented as

$$
\begin{aligned}
\xi_{k} & =\bar{F}_{k} \xi_{k-1}+\bar{B}_{k} u_{k}+\bar{w}_{k}, \\
\xi_{k} & =\operatorname{cat}\left\{x_{k}, y_{k}, \cdots, y_{k-d_{\max }}\right\} \\
y_{k} & =\operatorname{cat}\left\{y_{k}^{i}\right\}_{i=1}^{m}, \\
\bar{w}_{k} & =\operatorname{cat}\left\{w_{k}, H_{k} w_{k}+v_{k}, 0\right\} \\
H_{k} & =\operatorname{cat}\left\{H_{k}^{i}\right\}_{i=1}^{m}
\end{aligned}
$$

with the matrix coefficients

$$
\bar{F}_{k}=\left[\begin{array}{ccccc}
F_{k} & 0 & \cdots & 0 & 0 \\
H_{k} F_{k} & 0 & \cdots & 0 & 0 \\
0 & I_{n_{y}} & \cdots & 0 & 0 \\
\vdots & \vdots & \ddots & \vdots & \vdots \\
0 & 0 & \cdots & I_{n_{y}} & 0
\end{array}\right], \bar{B}_{k}=\left[\begin{array}{c}
B_{k} \\
H_{k} B_{k} \\
0 \\
\vdots \\
0
\end{array}\right]
$$

and the covariance matrix for $\bar{w}_{k}$ given by

$$
\mathbb{E}\left\{\bar{w}_{k} \bar{w}_{k}^{T}\right\}=\operatorname{diag}\left\{\left[\begin{array}{cc}
Q_{k} & Q_{k} H_{k}^{T} \\
H_{k} Q_{k} & R_{k}+H_{k} Q_{k} H_{k}^{T}
\end{array}\right], 0\right\} .
$$

The set of samples that are received by the estimator at $t$ can be concatenated to a vector $y_{k}^{s}$ defined as

$$
y_{k}^{s}=\operatorname{cat}\left\{y_{k-j}^{i}\right\}_{(j, i) \in \mathcal{J}_{k}}
$$

with $\mathcal{J}_{k}$ in (14) which can be written as

$$
\begin{aligned}
& y_{k}^{s}=\bar{H}_{k} \xi_{k}, \\
& \bar{H}_{k}=\left[\begin{array}{ll}
0 & \operatorname{diag}\left\{\bar{I}_{i}\right\}_{(j, i) \in \mathcal{J}_{k}}
\end{array}\right] \\
& \bar{I}_{i}=\left[\begin{array}{lll}
0_{n_{i} \times \sum_{j=1}^{i-1} n_{j}} & I_{n_{i}} & 0_{n_{i} \times \sum_{j=i+1}^{m} n_{j}}
\end{array}\right]
\end{aligned}
$$

The system (17a) with output $y_{k}^{s}$ in (21a) is a delay-free system and the ordinary Kalman filter in the appendix can be applied to estimate $\xi_{k}$ in (17b) which includes $x_{k}$. As a result, the estimation $\hat{x}_{k \mid k}$ in (4) is obtained as

$$
\hat{x}_{k \mid k}=\left[\begin{array}{llll}
I & 0 & \cdots & 0
\end{array}\right] \hat{\xi}_{k \mid k}
$$

\section{NumERICAL EXAMPLE}

In this section the proposed filtering methods are applied to an example system and their computational loads are compared. For this end, the time-dicretized state space model for a chain of $n_{x}$ integrators is considered as

$$
\begin{aligned}
x_{k+1} & =F x_{k}+B u_{k}+w_{k} \\
y_{k} & =H x_{k}+v_{k} \\
F & =e^{F_{c} h}, \quad B=\int_{0}^{h} e^{F_{c} \tau} B_{c} d \tau \\
F_{c} & =\left[\begin{array}{cc}
0 & I_{n_{x}-1} \\
0 & 0
\end{array}\right], B_{c}=\left[\begin{array}{l}
0 \\
1
\end{array}\right], H=\left[\begin{array}{ll}
1 & 0
\end{array}\right]
\end{aligned}
$$

in which $x_{k} \in \mathbb{R}^{n_{x}}$ is the vector of integrator outputs, $u_{k} \in \mathbb{R}$ is the input, $h=0.05, w_{k} \in \mathbb{R}^{n_{x}}$ and $v_{k} \in \mathbb{R}$ are uncorrelated Gaussian white noises with $\mathbb{E}\left\{w_{k} w_{k}^{T}\right\}=0.1 I$ and $\mathbb{E}\left\{v_{k}^{2}\right\}=$ 0.1 . To stabilize the system, a linear quadratic (LQR) state feedback controller $u_{k}=K x_{k}$ is applied which minimizes the quadratic cost function $J=\sum_{0}^{\infty}\left(x_{k}^{T} x+u_{k}^{2}\right)$.

The backward renovation filter (BRF) in section III and the augmented system filter (AGF) in section IV are equivalent and produce the same result, since they both generate the state estimation in (4). The simulation results for the state $x_{k}$ of the system (23) and the corresponding estimation $\hat{x}_{k \mid k}$ using either of the two filters are plotted in Fig. 1a through Fig. 1c. The delays $d_{k}$ are generated randomly with $\mathrm{P}\left\{d_{k}=\infty\right\}=0.05$, and equal probabilities $\mathrm{P}\left\{d_{k}=i\right\}$ for $i \in\left\{0 . . d_{\max }\right\}$ with $d_{\max }=20$. The delay values are plotted in Fig. 1d in which the missing measurements are indicated by a $\triangle$ symbol at the corresponding time step with zero height.

The computational loads of the two filtering methods are compared in terms of the average count of floating point operations (flops) per time step during a simulation with 300 time steps. The flop counts are calculated by summing the flop counts for individual matrix operations required by each method. The comparison is made over $n_{x} \in\{3,10,30\}$, $d_{\text {max }} \in\{5,10,20,50\}$ and the results are presented in Table I. According to the results, the load of computations increases with $n_{x}$ and $d_{\max }$ for both methods. However, the increase of flop count with respect to $n_{x}$ is much faster in the case of BRF method. On the other hand, the flop count increases more rapidly with the increase of $d_{\max }$ in the case of the AGF 


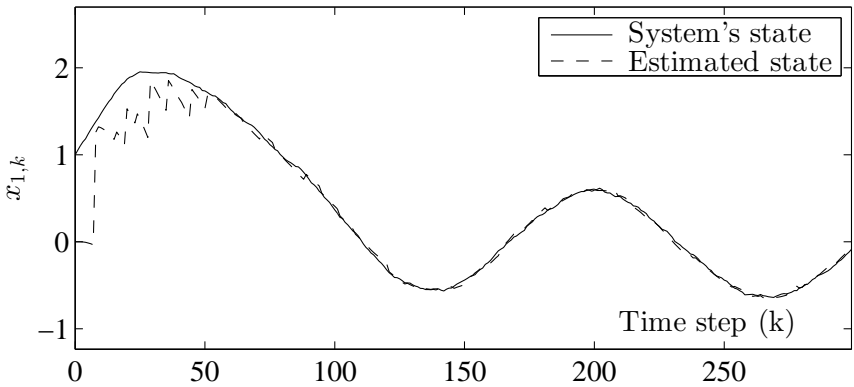

(a) First state variable.

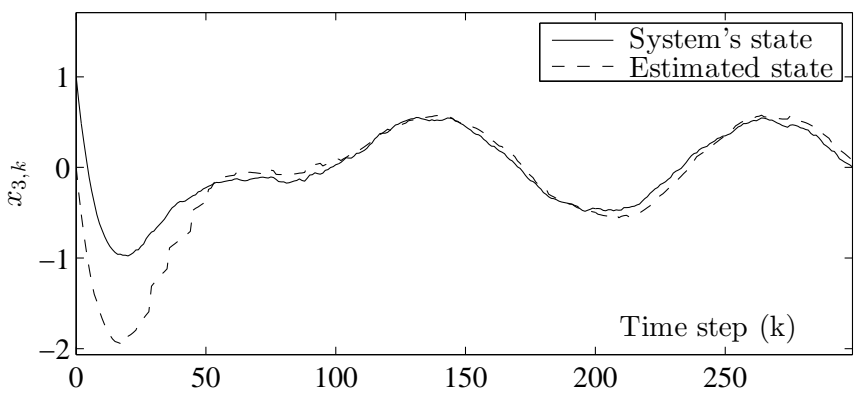

(c) Third state variable.

Fig. 1. Simulation of the methods for Kalman filtering with delays.

TABLE I

FLOP COUNTS OF BACKWARD RENOVATION FILTER (BRF) AND AUGMENTED SYSTEM FILTER (AGF) FOR DIFFERENT CONDITIONS.

\begin{tabular}{|c|c|c|c|c|c|}
\cline { 3 - 6 } \multicolumn{2}{c|}{} & \multicolumn{4}{c|}{ Flop count } \\
\hline \multirow{2}{*}{3} & Method & $d_{\max }=5$ & $d_{\max }=10$ & $d_{\max }=20$ & $d_{\max }=50$ \\
\cline { 2 - 6 } & BRF & $1.52 \times 10^{5}$ & $2.33 \times 10^{5}$ & $3.89 \times 10^{5}$ & $6.76 \times 10^{5}$ \\
\cline { 2 - 6 } & AGF & $6.86 \times 10^{5}$ & $2.23 \times 10^{6}$ & $9.94 \times 10^{6}$ & $5.63 \times 10^{7}$ \\
\hline \multirow{2}{*}{10} & BRF & $4.59 \times 10^{6}$ & $7.11 \times 10^{6}$ & $1.21 \times 10^{7}$ & $2.17 \times 10^{7}$ \\
\cline { 2 - 6 } & AGF & $3.21 \times 10^{6}$ & $6.83 \times 10^{6}$ & $2.06 \times 10^{7}$ & $8.66 \times 10^{7}$ \\
\hline \multirow{2}{*}{30} & BRF & $1.18 \times 10^{8}$ & $1.89 \times 10^{8}$ & $3.08 \times 10^{8}$ & $5.53 \times 10^{8}$ \\
\cline { 2 - 6 } & AGF & $3.17 \times 10^{7}$ & $4.61 \times 10^{7}$ & $8.67 \times 10^{7}$ & $2.28 \times 10^{8}$ \\
\hline
\end{tabular}

method. Therefore, it is advisable to use the BRF method in the case of long delays and to use the AGF method for systems with larger dimensionality.

\section{CONCLUSION}

Two existing approaches to Kalman filtering with delays have been extended to equivalent methods for optimal estimation in presence of multiple effects. These effects include timevarying delays in multiple measurement channels, missing measurements (e.g. due to packet losses or multirate sampling), and miss-ordered arrival of data. The extension of first approach which is based on recalculation of the past filtering variables has been presented as an algorithm using finite length memory buffers. The second approach which is based on system augmentation has been also extended to tackle missing measurements and the other effects. The computational loads

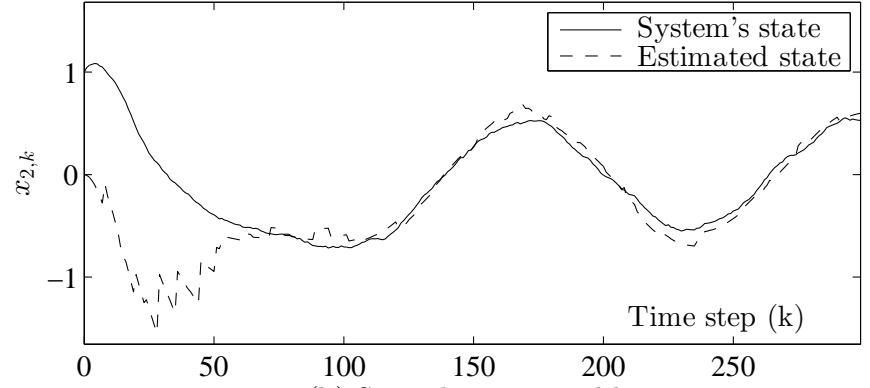

(b) Second state variable.

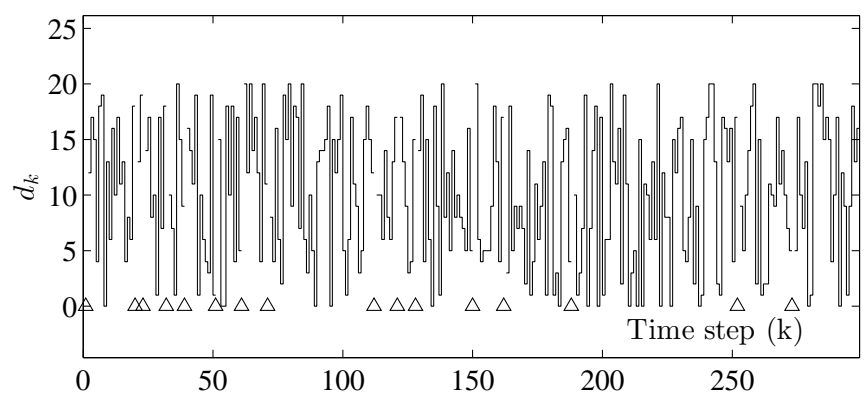

(d) Transmission delay.

of the resulting equivalent filtering methods were compared in terms of the flop counts for filtering integrator chains of various lengths. The comparison results suggest that the first method performs better for long delays while the second method can be more efficient for larger systems.

\section{APPENDIX A}

KALMAN FILTER FOR LINEAR SYSTEMS WITH VARYING OUTPUT DIMENSIONALITY

Consider a dynamical system system described by

$$
\begin{aligned}
& x_{k}=F_{k} x_{k-1}+B_{k} u_{k}+w_{k} \\
& y_{k}=H_{k} x_{k}+v_{k}^{i}
\end{aligned}
$$

with $x_{k} \in \mathbb{R}^{n_{x}}, u_{k} \in \mathbb{R}^{n_{u}}$, and $y_{k} \in \mathbb{R}^{n_{k}}$ where $n_{k}$ varies with $k$, and uncorrelated, zero-mean white Gaussian noise vectors $w_{k} \in \mathbb{R}^{n_{w}}$ and $v_{k} \in \mathbb{R}^{n_{k}}$ with $R_{k}=\mathbb{E}\left\{v_{k}^{i} v_{k}^{i T}\right\}$ and $Q_{k}=$ $\mathbb{E}\left\{w_{k} w_{k}^{T}\right\}$. The optimal estimation of $x_{k}$ is defined as

$$
\hat{x}_{k \mid k}=\mathbb{E}\left\{x_{k} \mid \mathcal{I}_{k}\right\}
$$

with $\mathcal{I}_{k}=\left\{y_{i} \mid i \leq k\right\}$. Given $\hat{x}_{0 \mid 0}$ and $P_{0 \mid 0}$, it is straightforward to show that the estimation $\hat{x}_{k \mid k}$ can be calculated recursively according to

$$
\begin{aligned}
& \hat{x}_{k \mid k-1}=F_{k} \hat{x}_{k-1 \mid k-1}+B_{k} u_{k}, \\
& P_{k \mid k-1}=F_{k} P_{k-1 \mid k-1} F_{k}^{T}+Q_{k}, \\
& K_{k}=P_{k \mid k-1} H_{k}^{T}\left(H_{k} P_{k \mid k-1} H_{k}^{T}+R_{k}\right)^{-1}, \\
& \hat{x}_{k \mid k}=\hat{x}_{k \mid k-1}+K_{k}\left(y_{k}-H_{k} \hat{x}_{k \mid k-1}\right), \\
& P_{k \mid k}=\left(I-K_{k} H_{k}\right) P_{k \mid k-1} .
\end{aligned}
$$

If $n_{k}=0$ for some $k$, then $H_{k}$ and $K_{k}$ in (26) become $0 \times n_{x}$ and $n_{x} \times 0$ empty matrices respectively, and the product $K_{k} H_{k}$ in (26e) is defined to be a $n_{x} \times n_{x}$ zero matrix. 


\section{REFERENCES}

[1] X. Zhang, Q. Han, and X. Yu, "Survey on recent advances in networked control systems," IEEE Transactions on Industrial Informatics, vol. 12, no. 5, pp. 1740-1752, Oct 2016.

[2] Lin Xiao, A. Hassibi, and J. P. How, "Control with random communication delays via a discrete-time jump system approach," in Proceedings of the 2000 American Control Conference. ACC (IEEE Cat. No.00CH36334), vol. 3, June 2000, pp. 2199-2204 vol.3.

[3] B. Sinopoli, L. Schenato, M. Franceschetti, K. Poolla, M. I. Jordan, and S. S. Sastry, "Kalman filtering with intermittent observations," IEEE Transactions on Automatic Control, vol. 49, no. 9, pp. 1453-1464, Sep. 2004.

[4] X. Liu and A. Goldsmith, "Kalman filtering with partial observation losses," in 2004 43rd IEEE Conference on Decision and Control (CDC)(IEEE Cat. No. 04CH37601), vol. 4. IEEE, 2004, pp. 41804186.

[5] L. Schenato, B. Sinopoli, M. Franceschetti, K. Poolla, and S. S. Sastry, "Foundations of control and estimation over lossy networks," Proceedings of the IEEE, vol. 95, no. 1, pp. 163-187, 2007.

[6] Y. Mo and B. Sinopoli, "Kalman filtering with intermittent observations: Tail distribution and critical value," IEEE Transactions on Automatic Control, vol. 57, no. 3, pp. 677-689, March 2012.

[7] H. L. Alexander, "State estimation for distributed systems with sensing delay," in Data Structures and Target Classification, V. Libby, Ed., vol. 1470, International Society for Optics and Photonics. SPIE, 1991, pp. $103-111$.

[8] T. D. Larsen, N. A. Andersen, O. Ravn, and N. K. Poulsen, "Incorporation of time delayed measurements in a discrete-time kalman filter," in Proceedings of the 37th IEEE Conference on Decision and Control (Cat. No.98CH36171), vol. 4, Dec 1998, pp. 3972-3977 vol.4.

[9] L. Schenato, "Optimal estimation in networked control systems subject to random delay and packet drop," IEEE Transactions on Automatic Control, vol. 53, no. 5, pp. 1311-1317, June 2008.

[10] S. Sun and G. Wang, "Modeling and estimation for networked systems with multiple random transmission delays and packet losses," Systems \& Control Letters, vol. 73, pp. 6 - 16, 2014.

[11] A. Fatehi and B. Huang, "Kalman filtering approach to multi-rate information fusion in the presence of irregular sampling rate and variable measurement delay," Journal of Process Control, vol. 53, pp. 15 - 25, 2017.

[12] I. Penarrocha, R. Sanchis, and J. Romero, "State estimator for multisensor systems with irregular sampling and time-varying delays," International Journal of Systems Science, vol. 43, no. 8, pp. 1441-1453, 2012.

[13] I. Penarrocha, R. Sanchis, and P. Albertos, "Estimation in multisensor networked systems with scarce measurements and time varying delays," Systems \& Control Letters, vol. 61, no. 4, pp. 555 - 562, 2012.

[14] C. Zhu, Y. Xia, L. Xie, and L. Yan, "Optimal linear estimation for systems with transmission delays and packet dropouts," IET Signal Processing, vol. 7, pp. 814-823, December 2013.

[15] S. Sun, L. Xie, W. Xiao, and Y. C. Soh, "Optimal linear estimation for systems with multiple packet dropouts," Automatica, vol. 44, no. 5, pp. $1333-1342,2008$.

[16] A. Gopalakrishnan, N. S. Kaisare, and S. Narasimhan, "Incorporating delayed and infrequent measurements in extended kalman filter based nonlinear state estimation," Journal of Process Control, vol. 21, no. 1, pp. $119-129,2011$.

[17] F.-H. Hsiao and S.-T. Pan, "Robust kalman filter synthesis for uncertain multiple time-delay stochastic systems," J. Dyn. Sys., Meas., Control., vol. 118 , no. 4, pp. 803-808, 1996.

[18] C. E. de Souza, R. M. Palhares, and P. D. Peres, "Robust $H_{\infty}$ filter design for uncertain linear systems with multiple time-varying state delays," IEEE Transactions on Signal Processing, vol. 49, no. 3, pp. 569-576, 2001.

[19] E. Fridman and U. Shaked, "A new $H_{\infty}$ filter design for linear time delay systems," Signal Processing, IEEE Transactions on, vol. 49, pp. $2839-2843,122001$.

[20] X.-M. Zhang and Q.-L. Han, "Event-based $h$ filtering for sampled-data systems," Automatica, vol. 51, pp. 55 - 69, 2015.

[21] X. Zhang, Q. Han, and B. Zhang, "An overview and deep investigation on sampled-data-based event-triggered control and filtering for networked systems," IEEE Transactions on Industrial Informatics, vol. 13, no. 1, pp. 4-16, 2017.

[22] M. Mahmoud, L. Xie, and Y. Soh, "Robust kalman filtering for discrete state-delay systems," IEE Proceedings - Control Theory and Applications, vol. 147, pp. 613-618, November 2000.
[23] X. Lu, L. Xie, H. Zhang, and W. Wang, "Robust kalman filtering for discrete-time systems with measurement delay," IEEE Transactions on Circuits and Systems II: Express Briefs, vol. 54, no. 6, pp. 522-526, 2007.

[24] X.-M. Zhang and Q.-L. Han, "Robust $H_{\infty}$ filtering for a class of uncertain linear systems with time-varying delay," Automatica, vol. 44 , no. 1 , pp. $157-166,2008$. 